\title{
Convex combinations, barycenters and convex functions
}

\section{Zlatko Pavić}

\section{"Correspondence:}

Zlatko.Pavic@sfsb.hr

Mechanical Engineering Faculty in Slavonski Brod, University of Osijek,

Trg Ivane Brlić Mažuranić 2,

Slavonski Brod, 35000, Croatia

\begin{abstract}
The article first shows one alternative definition of convexity in the discrete case. The correlation between barycenters, Jensen's inequality and convexity is studied in the integral case. The Hermite-Hadamard inequality is also obtained as a consequence of a concept of barycenters. Some derived results are applied to the quasi-arithmetic means and especially to the power means.
\end{abstract}

MSC: 26A51;26D15; 28A10; 28A25

Keywords: convex combination; barycenter; convex function; Jensen's inequality; quasi-arithmetic mean

\section{Introduction}

Sets with the common barycenter are observed in geometry, mechanics dealing with mass densities and probability theory in the study of random variables. Development and application of the theory of convex functions also includes barycenters. The following result, expressed by the measure and integral, is the most commonly used.

'Let $A, B \subset \mathbb{R}$ be bounded closed intervals so that $A \subset B$ and $\mu$ be a finite measure on $B$ so that $0<\mu(A)<\mu(B)$. If the barycenter equality

$$
\frac{1}{\mu(A)} \int_{A} t d \mu(t)=\frac{1}{\mu(B)} \int_{B} t d \mu(t)
$$

is valid, then the inequality

$$
\frac{1}{\mu(A)} \int_{A} f(t) d \mu(t) \leq \frac{1}{\mu(B)} \int_{B} f(t) d \mu(t)
$$

holds for every $\mu$-integrable convex function $f: B \rightarrow \mathbb{R}$ :

The related problems with different types of measures and mathematical expectations were investigated in [1]. The inequality in (1.2) under the condition in (1.1) was extended in [2]. The intention of this paper is still more to connect the quoted implication (in the extended form) with convex functions, in the discrete and integral case. We also wanted to insert the quasi-arithmetic means into this implication.

The quoted result was actually observed in Banach spaces. So, it was assumed that $A$ and $B$ are bounded closed convex subsets of a Banach space $E$ such that $A \subset B$ and $f: B \rightarrow \mathbb{R}$ is a convex function. The opposite examples are found in [2] already for $E=\mathbb{R}^{2}$ and $E=\mathbb{R}^{3}$.

\section{Springer}

(c) 2013 Pavić; licensee Springer. This is an Open Access article distributed under the terms of the Creative Commons Attribution License (http://creativecommons.org/licenses/by/2.0), which permits unrestricted use, distribution, and reproduction in any medium, provided the original work is properly cited. 
Throughout the whole paper, we suppose that $I \subseteq \mathbb{R}$ is a non-degenerate interval. Subintervals from $I$ will also be non-degenerate. Convex hull of a set $X$ will be denoted by $\operatorname{co} X$.

The main results of the paper are presented in Sections 2 and 3.

\section{Convex combinations with convex functions}

In this section, we show the connection between the convex combinations and the convex functions. The basic form of Jensen's inequality is obtained using the assumption of the equality of convex combinations. An alternative definition of convexity is also presented.

Throughout the section, we will assume that $n$ is a positive number greater than or equal to 2 , i.e., $n \geq 2$.

An elementary mean of points $x_{1}, \ldots, x_{n} \in I$ is the arithmetic mean $\frac{1}{n} \sum_{i=1}^{n} x_{i} \in I$. A discrete generalization of the arithmetic mean is the convex combination or the weighted mean $\sum_{i=1}^{n} p_{i} x_{i} \in I$ with coefficients $p_{i} \in[0,1]$ such that $\sum_{i=1}^{n} p_{i}=1$.

Theorem A Let $x_{1}, \ldots, x_{n} \in I$ be points such that

$$
x_{i} \notin \operatorname{co}\left\{x_{1}, \ldots, x_{k}\right\} \quad \text { for } i=k+1, \ldots, n, \text { where } 1 \leq k \leq n-1 .
$$

Let $\alpha_{1}, \ldots, \alpha_{n} \in \mathbb{R}$ be non-negative numbers such that

$$
0<\sum_{i=1}^{k} \alpha_{i}=\alpha<\beta=\sum_{i=1}^{n} \alpha_{i}
$$

If one of the equalities

$$
\frac{1}{\alpha} \sum_{i=1}^{k} \alpha_{i} x_{i}=\frac{1}{\beta} \sum_{i=1}^{n} \alpha_{i} x_{i}=\frac{1}{\beta-\alpha} \sum_{i=k+1}^{n} \alpha_{i} x_{i}
$$

is valid, then the double inequality

$$
\frac{1}{\alpha} \sum_{i=1}^{k} \alpha_{i} f\left(x_{i}\right) \leq \frac{1}{\beta} \sum_{i=1}^{n} \alpha_{i} f\left(x_{i}\right) \leq \frac{1}{\beta-\alpha} \sum_{i=k+1}^{n} \alpha_{i} f\left(x_{i}\right)
$$

holds for every convex function $f: I \rightarrow \mathbb{R}$.

Theorem A was realized in [2, Proposition 2]. The proof of Theorem A can be done by direct application of convexity on the model of the proof in [2, Proposition 1] with the chord line (the line through points $T_{1}(a, f(a))$ and $T_{2}(b, f(b))$ of the graph of $f$ )

$$
f_{[a, b]}^{c h o}(x)=\frac{b-x}{b-a} f(a)+\frac{x-a}{b-a} f(b)
$$

using $a=\min \left\{x_{1}, \ldots, x_{k}\right\}$ and $b=\max \left\{x_{1}, \ldots, x_{k}\right\}$ and by putting the sums instead of integrals. So, the implication of Theorem A can be proved without applying the basic Jensen inequality. 
Corollary 2.1 Let $x_{1}, \ldots, x_{n} \in I$ and $\alpha_{1}, \ldots, \alpha_{n} \in \mathbb{R}$ be as in Theorem A with the additional condition

$$
\sum_{i=1}^{k} \alpha_{i}=\sum_{i=k+1}^{n} \alpha_{i}
$$

If the equality

$$
\sum_{i=1}^{k} \alpha_{i} x_{i}=\sum_{i=k+1}^{n} \alpha_{i} x_{i}
$$

is valid, then the inequality

$$
\sum_{i=1}^{k} \alpha_{i} f\left(x_{i}\right) \leq \sum_{i=k+1}^{n} \alpha_{i} f\left(x_{i}\right)
$$

holds for every function $f: I \rightarrow \mathbb{R}$ which satisfies the implication of Theorem A.

The next consequence is the basic form of Jensen's inequality, as the main result in this section.

Theorem 2.2 If $\sum_{i=1}^{n} p_{i} x_{i}$ is a convex combination of points $x_{i} \in I$ with coefficients $p_{i} \in$ $[0,1]$ so that $\sum_{i=1}^{n} p_{i}=1$, then the inequality

$$
f\left(\sum_{i=1}^{n} p_{i} x_{i}\right) \leq \sum_{i=1}^{n} p_{i} f\left(x_{i}\right)
$$

holds for every function $f: I \rightarrow \mathbb{R}$ which satisfies the implication of Theorem A.

Proof Let $x_{0}=\sum_{i=1}^{n} p_{i} x_{i}$ with $\sum_{i=1}^{n} p_{i}=1$. Without loss of generality, suppose that all $x_{i}$ are pairwise different and all $p_{i}>0$.

If $x_{0} \neq x_{i}$ for all $i$, then we apply Corollary 2.1 to the sets of points $\left\{x_{0}\right\}$ and $\left\{x_{1}, \ldots, x_{n}\right\}$ with associated coefficients $\alpha_{0}=1$ and $\alpha_{i}=p_{i}$ for $i=1, \ldots, n$. It follows

$$
f\left(\sum_{i=1}^{n} p_{i} x_{i}\right)=f\left(x_{0}\right) \leq \sum_{i=1}^{n} p_{i} f\left(x_{i}\right)
$$

If $x_{0}=x_{i_{0}}$ for some $i=i_{0}$, then

$$
x_{0}=\frac{1}{1-p_{i_{0}}} \sum_{\substack{i=1 \\ i \neq i_{0}}}^{n} p_{i} x_{i}
$$

is also the convex combination. Since $x_{0} \neq x_{i}$ for all $i \neq i_{0}$, we can apply the previous case. It follows

$$
f\left(\sum_{i=1}^{n} p_{i} x_{i}\right)=f\left(x_{0}\right) \leq \frac{1}{1-p_{i_{0}}} \sum_{\substack{i=1 \\ i \neq i_{0}}}^{n} p_{i} f\left(x_{i}\right),
$$


and therefore

$$
\left(1-p_{i_{0}}\right) f\left(x_{0}\right) \leq \sum_{i=1}^{n} p_{i} f\left(x_{i}\right)-p_{i_{0}} f\left(x_{i_{0}}\right)
$$

so we have

$$
f\left(x_{0}\right) \leq \sum_{i=1}^{n} p_{i} f\left(x_{i}\right)
$$

because $x_{i_{0}}=x_{0}$.

So, using Theorem A, we can derive the basic Jensen inequality. The previous results can be written in the following theorem as the alternative definition of convexity.

Theorem 2.3 A function $f: I \rightarrow \mathbb{R}$ is convex if and only if it satisfies the implication of Theorem A.

\section{Integral arithmetic means with convex functions}

In this section, we show the connection between the convexity and the barycenters. The integral form of Jensen's inequality for the measures which satisfy some conditions is obtained using the barycenters.

Integral generalizations of the concept of arithmetic mean in the finite measure spaces are the integral arithmetic mean or the barycenter of measurable set and the integral arithmetic mean of integrable function; see [3, p.44]. In particular, if we have a probabilistic measure, then the integral arithmetic mean of a random variable is just its mathematical expectation.

Let $\mu$ be a finite measure on $I$ and $A \subseteq I$ be a $\mu$-measurable set with $\mu(A)>0$. We define the $\mu$-barycenter of $A$ by

$$
\mathcal{B}(A, \mu)=\frac{1}{\mu(A)} \int_{A} t d \mu(t)
$$

If $f: I \rightarrow \mathbb{R}$ is a $\mu$-integrable function on $A$, then we define the $\mu$-arithmetic mean of $f$ on $A$ by

$$
\mathcal{M}(A, f, \mu)=\frac{1}{\mu(A)} \int_{A} f(t) d \mu(t) .
$$

Note that $\mathcal{M}\left(A, 1_{A}, \mu\right)=\mathcal{B}(A, \mu)$, where $1_{A}$ is an identity function on $A$. If $A$ is the interval, then its $\mu$-barycenter $\mathcal{B}(A, \mu)$ belongs to $A$. If $A$ is the interval and $f$ is continuous on $A$, then its $\mu$-arithmetic mean on $A$ belongs to $f(A)$.

Theorem B Let $\mu$ be a finite measure on I. Let $B \subseteq$ I be a $\mu$-measurable set and $A \subset B$ be a bounded interval such that

$$
0<\mu(A)<\mu(B) .
$$


If one of the equalities

$$
\mathcal{B}(A, \mu)=\mathcal{B}(B, \mu)=\mathcal{B}(B \backslash A, \mu)
$$

is valid, then the double inequality

$$
\mathcal{M}(A, f, \mu) \leq \mathcal{M}(B, f, \mu) \leq \mathcal{M}(B \backslash A, f, \mu)
$$

holds for every convex $\mu$-integrable function $f: I \rightarrow \mathbb{R}$.

The version of Theorem B for the bounded closed intervals $A$ and $B$ was proved in [2, Proposition 1] by using the chord line $y=f_{[a, b]}^{c h o}(x)$ when $A=[a, b]$. The proof was realized without applying the integral Jensen inequality. The same proof can be applied to Theorem B with $a=\inf A$ and $b=\sup A$.

It is unfortunate that Theorem $B$ is not valid for the convex functions of several variables. Such examples for the convex function of two and three variables are shown in [2, Example 1,2].

The next corollary is the generalization of Theorem B. It can be also useful in some applications, especially in applications on quasi-arithmetic means.

Corollary 3.1 Let $\mu$ be a finite measure on I. Let $g: I \rightarrow \mathbb{R}$ be a continuous $\mu$-integrable function and $J=g(I)$. Let $B \subseteq I$ be a $\mu$-measurable set and $A \subset B$ be a bounded interval such that

$$
0<\mu(A)<\mu(B)
$$

If one of the equalities

$$
\mathcal{M}(A, g, \mu)=\mathcal{M}(B, g, \mu)=\mathcal{M}(B \backslash A, g, \mu)
$$

is valid, then the double inequality

$$
\mathcal{M}(A, f \circ g, \mu) \leq \mathcal{M}(B, f \circ g, \mu) \leq \mathcal{M}(B \backslash A, f \circ g, \mu)
$$

holds for every convex function $f: J \rightarrow \mathbb{R}$ provided that $f \circ g$ is $\mu$-integrable.

The following is the integral analogy of Corollary 2.1 .

Corollary 3.2 Let $\mu$ and $A, B \subseteq I$ be as in Theorem B with the addition

$$
\mu(A)=\mu(B \backslash A) .
$$

If the equality

$$
\int_{A} t d \mu(t)=\int_{B \backslash A} t d \mu(t)
$$


is valid, then the inequality

$$
\int_{A} f(t) d \mu(t) \leq \int_{B \backslash A} f(t) d \mu(t)
$$

holds for every $\mu$-integrable function $f: I \rightarrow \mathbb{R}$ which satisfies the implication of Theorem $\mathrm{B}$.

The concept of barycenter enables the realization of the most important inequalities such as the Jensen inequality and the Hermite-Hadamard inequality. This approach requires fine measures.

A measure $\mu$ on $I$ is said to be continuous if $\mu(\{t\})=0$ for every point $t \in I$. Take an interval $[a, b] \subseteq I$. If $\mu$ is a continuous finite measure on $I$, then the functions

$$
x \mapsto \mu([a, x]) \text { and } \quad x \mapsto \int_{[a, x]} t d \mu(t)
$$

are continuous and monotone on $[a, b]$. If additionally the measure $\mu$ is positive on the intervals from $I$, then the above functions are strictly monotone.

In the rest of this section, we will use the continuous finite measure on $I$ which is positive on the intervals from $I$, that is, $\mu(S)>0$ for every interval $S \subseteq I$.

Lemma 3.3 Let $\mu$ be a continuous finite measure on I which is positive on the intervals from $I$.

If a is a point from the interior of $I$, then a decreasing series $\left(A_{n}\right)_{n}$ of intervals $A_{n} \subseteq I$ exists so that

$$
\bigcap_{n=1}^{\infty} A_{n}=\{a\} \quad \text { and } \mathcal{B}\left(A_{n}, \mu\right)=a .
$$

Proof Take a point $a$ from the interior of $I$.

In the first step, we choose points $x_{1}, y_{1} \in I$ such that $x_{1}<a<y_{1}$ and determine the $\mu$ barycenter of the interval $\left[x_{1}, y_{1}\right]$ :

$$
a_{1}=\frac{1}{\mu\left(\left[x_{1}, y_{1}\right]\right)} \int_{\left[x_{1}, y_{1}\right]} t d \mu(t) .
$$

If $a_{1}=a$, then we take $A_{1}=\left[x_{1}, y_{1}\right]$. If $a_{1}>a$, then we observe the function $g:\left[a, y_{1}\right] \rightarrow \mathbb{R}$ defined by

$$
g(y)=\frac{1}{\mu\left(\left[x_{1}, y\right]\right)} \int_{\left[x_{1}, y\right]} t d \mu(t)-a .
$$

Since $g$ is continuous, $g(a)<0$ and $g\left(y_{1}\right)>0$, there must be a point $\bar{y}_{1} \in\left\langle a, y_{1}\right\rangle$ such that $g\left(\bar{y}_{1}\right)=0$. In this case we can take $A_{1}=\left[x_{1}, \bar{y}_{1}\right]$. If $a_{1}<a$, then we increase $x_{1}$ until we obtain one of the previous two cases.

In the next step, if $A_{1}=\left[x_{1}, y_{1}\right]$, we take points

$$
x_{2}=\frac{x_{1}+a}{2} \text { and } y_{2}=\frac{a+y_{1}}{2} \text {, }
$$

and repeat the previous procedure to determine $A_{2}$. 
Remark 3.4 The function $x \mapsto \mu\left(\left[x, y_{x}\right]\right)$, where $y_{x}$ is defined by

$$
\frac{1}{\mu\left(\left[x, y_{x}\right]\right)} \int_{\left[x, y_{x}\right]} t d \mu(t)=a,
$$

is strictly decreasing continuous on $\left[x_{1}, a\right\rangle$ with $\lim _{x \rightarrow a-} \mu\left(\left[x, y_{x}\right]\right)=0$.

The following consequence is the integral form of Jensen's inequality, as the main result in this section.

Theorem 3.5 Let $\mu$ be a continuous finite measure on I which is positive on the intervals from $I$.

If $B \subseteq I$ is a union of intervals, then the inequality

$$
f\left(\frac{1}{\mu(B)} \int_{B} t d \mu(t)\right) \leq \frac{1}{\mu(B)} \int_{B} f(t) d \mu(t)
$$

holds for every continuous $\mu$-integrable function $f: I \rightarrow \mathbb{R}$ which satisfies the implication of Theorem $B$ for unions $B$ of intervals from $I$ and bounded intervals $A \subset B$.

Proof Let $B \subseteq I$ be a union of intervals and let

$$
a=\frac{1}{\mu(B)} \int_{B} t d \mu(t)
$$

be its $\mu$-barycenter. We observe three cases depending on the $\mu$-barycenter $a$.

If $a$ belongs to the interior of $B$, then using the procedure described in Lemma 3.3, we can determine a decreasing series $\left(A_{n}\right)_{n}$ of intervals $A_{n} \subset B$ so that

$$
\bigcap_{n=1}^{\infty} A_{n}=\{a\}
$$

and

$$
\mathcal{B}\left(A_{n}, \mu\right)=\frac{1}{\mu\left(A_{n}\right)} \int_{A_{n}} t d \mu(t)=a \text { for every } A_{n} .
$$

We have

$$
\frac{1}{\mu\left(A_{n}\right)} \int_{A_{n}} t d \mu(t)=\frac{1}{\mu(B)} \int_{B} t d \mu(t),
$$

and since $\mu$-integrable function $f$ satisfies the implication of Theorem $\mathrm{B}$, from the lefthand side of the inequality in (3.4), we get

$$
\frac{1}{\mu\left(A_{n}\right)} \int_{A_{n}} f(t) d \mu(t) \leq \frac{1}{\mu(B)} \int_{B} f(t) d \mu(t) .
$$

After allowing $n \rightarrow \infty$, since $f$ is continuous, we get

$$
f\left(\frac{1}{\mu(B)} \int_{B} t d \mu(t)\right)=f(a)=\lim _{n \rightarrow \infty} \frac{1}{\mu\left(A_{n}\right)} \int_{A_{n}} f(t) d \mu(t) \leq \frac{1}{\mu(B)} \int_{B} f(t) d \mu(t)
$$

which ends the proof of this case. 
If $a$ is the boundary point of $B$, then we take small $\varepsilon>0$ and put $B_{\varepsilon}=[a-\varepsilon, a] \cup B$ or $B_{\varepsilon}=[a, a+\varepsilon] \cup B$. It provides that $\mu$-barycenter $a_{\varepsilon}$ of $B_{\varepsilon}$ belongs to the interior of $B_{\varepsilon}$. First, we apply the above procedure to $B_{\varepsilon}$ and its $\mu$-barycenter $a_{\varepsilon}$, and after that allow $\varepsilon \rightarrow 0$.

If $a$ does not belong to $B$ and if $a$ is not the boundary point of $B$, then we take small $\varepsilon>0$ and put $B_{\varepsilon}=[a-\varepsilon, a+\varepsilon] \cup B$. It provides that $\mu$-barycenter $a_{\varepsilon}$ of $B_{\varepsilon}$ belongs to the interior of $B_{\varepsilon}$. We apply the procedure from the first case to $B_{\varepsilon}$, and after that let $\varepsilon \rightarrow 0$.

Remark 3.6 The function $f$ from Corollary 3.5 must be continuous; otherwise, it may happen

$$
\lim _{n \rightarrow \infty} \frac{1}{\mu\left(A_{n}\right)} \int_{A_{n}} f(t) d \mu(t)=\lim _{n \rightarrow \infty} f\left(a_{n}\right) \neq f(a),
$$

where the series $\left(a_{n}\right)_{n}$ of the $\mu$-barycenters $a_{n}$ of intervals $A_{n}$ converges to $a$.

Thus, using Theorem B, we can realize the integral form of Jensen's inequality for continuous functions, unions of intervals and continuous finite measures which are positive on intervals. The following is the equivalent connection between convexity and Theorem B.

Theorem 3.7 Let $\mu$ be a continuous finite measure on I which is positive on the intervals from $I$. A continuous $\mu$-integrable function $f: I \rightarrow \mathbb{R}$ is convex if and only if it satisfies the implication of Theorem $\mathrm{B}$ for finite unions $B$ of intervals from I and bounded intervals $A \subset B$.

Proof The necessity follows from Theorem B. Let us prove the sufficiency on the interior of $I$. Take any convex combination $p x+q y$ of two different points $x$ and $y$ from the interior of $I$ with the positive coefficients $p$ and $q$. Suppose $x<y$.

The basic idea of the proof is to determine the small intervals $B_{n}^{x}$ and $B_{n}^{y}$ with the barycenters $x$ and $y$ such that $\mu\left(B_{n}^{x}\right) / \mu\left(B_{n}^{y}\right)=p / q$. Suppose we have $B_{n}^{x}$ and $B_{n}^{y}$ with barycenters $x$ and $y$. If $\mu\left(B_{n}^{x}\right) / \mu\left(B_{n}^{y}\right)>p / q$, then we decrease $B_{n}^{x}$. If $\mu\left(B_{n}^{x}\right) / \mu\left(B_{n}^{y}\right)<p / q$, then we decrease $B_{n}^{y}$.

Using the procedure from Lemma 3.3, we can determine the decreasing series $\left(B_{n}^{x}\right)_{n}$, $\left(A_{n}\right)_{n},\left(B_{n}^{y}\right)_{n}$ of pairwise disjoint intervals $B_{n}^{x}, A_{n}, B_{n}^{y}$ from $I$ satisfying the following conditions:

$$
\begin{array}{ll}
\bigcap_{n=1}^{\infty} B_{n}^{x}=\{x\}, \quad \bigcap_{n=1}^{\infty} A_{n}=\{p x+q y\}, & \bigcap_{n=1}^{\infty} B_{n}^{y}=\{y\}, \\
\mathcal{B}\left(B_{n}^{x}, \mu\right)=x, \quad \mathcal{B}\left(A_{n}, \mu\right)=p x+q y, \quad \mathcal{B}\left(B_{n}^{y}, \mu\right)=y, \\
\frac{\mu\left(B_{n}^{x}\right)}{\mu\left(B_{n}^{x} \cup B_{n}^{y}\right)}=p, \quad \mu\left(A_{n}\right)=\mu\left(B_{n}^{x} \cup B_{n}^{y}\right), \quad \frac{\mu\left(B_{n}^{y}\right)}{\mu\left(B_{n}^{x} \cup B_{n}^{y}\right)}=q .
\end{array}
$$

If

$$
B_{n}=B_{n}^{x} \cup A_{n} \cup B_{n}^{y},
$$


then it follows

$$
\begin{aligned}
\mathcal{B}\left(A_{n}, \mu\right) & =p x+q y=\frac{1}{\mu\left(B_{n} \backslash A_{n}\right)} \int_{B_{n}^{x}} t d \mu(t)+\frac{1}{\mu\left(B_{n} \backslash A_{n}\right)} \int_{B_{n}^{y}} t d \mu(t) \\
& =\frac{1}{\mu\left(B_{n} \backslash A_{n}\right)} \int_{B_{n} \backslash A_{n}} t d \mu(t)=\mathcal{B}\left(B_{n} \backslash A_{n}, \mu\right) .
\end{aligned}
$$

Applying the inequality in (3.4) from Theorem B, we have

$$
\begin{aligned}
\mathcal{M}\left(A_{n}, f, \mu\right) & \leq \mathcal{M}\left(B_{n} \backslash A_{n}, f, \mu\right) \\
& =\frac{1}{\mu\left(B_{n} \backslash A_{n}\right)} \int_{B_{n}^{x}} f(t) d \mu(t)+\frac{1}{\mu\left(B_{n} \backslash A_{n}\right)} \int_{B_{n}^{y}} f(t) d \mu(t) \\
& =p \mathcal{M}\left(B_{n}^{x}, f, \mu\right)+q \mathcal{M}\left(B_{n}^{y}, f, \mu\right),
\end{aligned}
$$

and letting $n \rightarrow \infty$, since $f$ is continuous, we obtain

$$
f(p x+q y) \leq p f(x)+q f(y)
$$

which ends the proof.

For details on global bounds for generalized Jensen's inequality, see [4].

The Hermite-Hadamard inequality is also the consequence of Theorem B.

Corollary 3.8 Let $\mu$ be a continuous finite measure on I which is positive on the intervals from $I$.

$$
\text { If }[a, b] \subseteq I \text { and }
$$

$$
p a+q b=\frac{1}{\mu([a, b])} \int_{[a, b]} t d \mu(t)
$$

then the inequality

$$
f(p a+q b) \leq \frac{1}{\mu([a, b])} \int_{[a, b]} f(t) d \mu(t) \leq p f(a)+q f(b)
$$

holds for every continuous function $f: I \rightarrow \mathbb{R}$ which satisfies the implication of Theorem $\mathrm{B}$ for finite unions $B$ of intervals from $I$ and bounded intervals $A \subset B$.

Proof Let us prove the corollary when $[a, b]$ belongs to the interior of $I$. Let $\left(B_{n}^{a}\right)_{n},\left(A_{n}\right)_{n}$, $\left(B_{n}^{b}\right)_{n}$ be the decreasing series of pairwise disjoint intervals $B_{n}^{a}, A_{n}, B_{n}^{b}$ from $I$ as in Theorem 3.7, with $a$ instead of $x$ and $b$ instead of $y$. Let us introduce also the increasing series $\left(\bar{A}_{n}\right)_{n}$ of intervals $\bar{A}_{n}$ so that

$$
A_{n} \subset \bar{A}_{n}, \quad \bar{A}_{n} \cap\left(B_{n}^{a} \cup B_{n}^{b}\right)=\emptyset, \quad \bigcup_{n=1}^{\infty} \bar{A}_{n}=[a, b] \quad \text { and } \quad \mathcal{B}\left(\bar{A}_{n}, \mu\right)=p a+q b .
$$

We construct an interval $\bar{A}_{n+1}$ by increasing the interval $\bar{A}_{n}$, after we have constructed intervals $B_{n+1}^{a}$ and $B_{n+1}^{b}$ by decreasing the intervals $B_{n}^{a}$ and $B_{n}^{b}$. 
If

$$
\bar{B}_{n}=B_{n}^{a} \cup \bar{A}_{n} \cup B_{n}^{b},
$$

then we have the barycenter equalities

$$
\mathcal{B}\left(A_{n}, \mu\right)=\mathcal{B}\left(\bar{A}_{n}, \mu\right)=\mathcal{B}\left(\bar{B}_{n} \backslash \bar{A}_{n}, \mu\right)=p a+q b .
$$

After applying the inequality in (3.4) to the pairs $A_{n}, \bar{A}_{n}$ and $\bar{A}_{n}, \bar{B}_{n} \backslash \bar{A}_{n}$, we get

$$
\mathcal{M}\left(A_{n}, f, \mu\right) \leq \mathcal{M}\left(\bar{A}_{n}, f, \mu\right) \leq \mathcal{M}\left(\bar{B}_{n} \backslash \bar{A}_{n}, f, \mu\right),
$$

that is,

$$
\frac{1}{\mu\left(A_{n}\right)} \int_{A_{n}} f(t) d \mu(t) \leq \frac{1}{\mu\left(\bar{A}_{n}\right)} \int_{\bar{A}_{n}} f(t) d \mu(t) \leq \frac{1}{\mu\left(\bar{B}_{n} \backslash \bar{A}_{n}\right)} \int_{\bar{B}_{n} \backslash \bar{A}_{n}} f(t) d \mu(t) .
$$

Letting $n \rightarrow \infty$, we obtain the inequality in (3.10).

An interesting version of the Hermite-Hadamard inequality in a non-positive curvature space was obtained in [5].

\section{Applications on quasi-arithmetic means}

In the applications of convexity, we often use strictly monotone continuous functions $\varphi, \psi: I \rightarrow \mathbb{R}$ such that $\psi$ is convex with respect to $\varphi$ ( $\psi$ is $\varphi$-convex), that is, $f=\psi \circ \varphi^{-1}$ is convex by [6, Definition 1.19]. A similar notation is used for concavity.

Let $x_{1}, \ldots, x_{n} \in I$ be points and $p_{1}, \ldots, p_{n} \in[0,1]$ be numbers such that $\sum_{i=1}^{n} p_{i}=1$. The discrete basic $\varphi$-quasi-arithmetic mean of points (particles) $x_{i}$ with coefficients (weights) $p_{i}$ is the point

$$
\mathcal{M}_{\varphi}\left(x_{i}, p_{i}\right)=\varphi^{-1}\left(\sum_{i=1}^{n} p_{i} \varphi\left(x_{i}\right)\right)
$$

which belongs to $I$ because $\sum_{i=1}^{n} p_{i} \varphi\left(x_{i}\right)$ belongs to $\varphi(I)$.

Theorem C Let $\varphi, \psi: I \rightarrow \mathbb{R}$ be strictly monotone continuous functions. Let $x_{1}, \ldots, x_{n} \in I$ be points such that

$$
x_{i} \notin \operatorname{co}\left\{x_{1}, \ldots, x_{k}\right\} \quad \text { for } i=k+1, \ldots, n, \text { where } 1 \leq k \leq n-1 .
$$

Let $\alpha_{1}, \ldots, \alpha_{n} \in \mathbb{R}$ be non-negative numbers such that

$$
0<\sum_{i=1}^{k} \alpha_{i}=\alpha<\beta=\sum_{i=1}^{n} \alpha_{i} .
$$

If $\psi$ is either $\varphi$-convex and increasing or $\varphi$-concave and decreasing, and if one of the equalities

$$
\mathcal{M}_{\varphi}\left(x_{i}, \frac{\alpha_{i}}{\alpha}\right)_{i=1}^{k}=\mathcal{M}_{\varphi}\left(x_{i}, \frac{\alpha_{i}}{\beta}\right)_{i=1}^{n}=\mathcal{M}_{\varphi}\left(x_{i}, \frac{\alpha_{i}}{\beta-\alpha}\right)_{i=k+1}^{n}
$$


is valid, then the double inequality

$$
\mathcal{M}_{\psi}\left(x_{i}, \frac{\alpha_{i}}{\alpha}\right)_{i=1}^{k} \leq \mathcal{M}_{\psi}\left(x_{i}, \frac{\alpha_{i}}{\beta}\right)_{i=1}^{n} \leq \mathcal{M}_{\psi}\left(x_{i}, \frac{\alpha_{i}}{\beta-\alpha}\right)_{i=k+1}^{n}
$$

holds.

If $\psi$ is either $\varphi$-convex and decreasing or $\varphi$-concave and increasing, then the reverse double inequality is valid in (4.3).

Theorem $\mathrm{C}$ was proved in [2, Corollary 1] by application of Theorem A. The application of Theorem $\mathrm{C}$ on the discrete basic power means can be found in [2, Corollary 2].

If $\mu$ is a finite measure on $I$ and $A \subseteq I$ is a measurable set with the positive measure, then we define the integral $\varphi$-quasi-arithmetic mean on the set $A$ with respect to the measure $\mu$ by

$$
\mathcal{M}_{\varphi}(A, \mu)=\varphi^{-1}\left(\frac{1}{\mu(A)} \int_{A} \varphi(t) d \mu(t)\right)
$$

If $A$ is the interval, then its $\varphi$-quasi-arithmetic mean $\mathcal{M}_{\varphi}(A, \mu)$ belongs to $A$ because the point $(1 / \mu(A)) \int_{A} \varphi(t) d \mu(t)$ belongs to $\varphi(A)$. If $A$ is not connected, then $\mathcal{M}_{\varphi}(A, \mu)$ may be outside of $A$.

Theorem 4.1 Let $\mu$ be a finite measure on $I$. Let $\varphi, \psi: I \rightarrow \mathbb{R}$ be strictly monotone continuous $\mu$-integrable functions. Let $B \subseteq I$ be a $\mu$-measurable set and $A \subset B$ be a bounded interval such that

$$
0<\mu(A)<\mu(B) .
$$

If $\psi$ is either $\varphi$-convex and increasing or $\varphi$-concave and decreasing, and if one of the equalities

$$
\mathcal{M}_{\varphi}(A, \mu)=\mathcal{M}_{\varphi}(B, \mu)=\mathcal{M}_{\varphi}(B \backslash A, \mu)
$$

is valid, then the double inequality

$$
\mathcal{M}_{\psi}(A, \mu) \leq \mathcal{M}_{\psi}(B, \mu) \leq \mathcal{M}_{\psi}(B \backslash A, \mu)
$$

holds.

If $\psi$ is either $\varphi$-convex and decreasing or $\varphi$-concave and increasing, then the reverse double inequality is valid in (4.6).

Proof Let us prove the case when $\psi$ is $\varphi$-convex and increasing. If we apply the function $\varphi$ to the equalities in (4.5), then it follows

$$
\frac{1}{\mu(A)} \int_{A} \varphi(t) d \mu(t)=\frac{1}{\mu(B)} \int_{B} \varphi(t) d \mu(t)=\frac{1}{\mu(B \backslash A)} \int_{B \backslash A} \varphi(t) d \mu(t) .
$$


Now, we can apply Corollary 3.1 with convex function $f=\psi \circ \varphi^{-1}$, and since $f(\varphi(t))=\psi(t)$, we have

$$
\frac{1}{\mu(A)} \int_{A} \psi(t) d \mu(t) \leq \frac{1}{\mu(B)} \int_{B} \psi(t) d \mu(t) \leq \frac{1}{\mu(B \backslash A)} \int_{B \backslash A} \psi(t) d \mu(t) .
$$

Finally, we apply the increasing function $\psi^{-1}$ to the above inequalities and get the double inequality in (4.6).

As a special case of the mean in (4.4) with $I=\left\langle 0,+\infty>, \varphi_{r}(t)=t^{r}\right.$ for $r \neq 0$ and $\varphi_{0}(t)=$ $\ln t$, we get the integral power mean on the set $A$ :

$$
\mathcal{M}_{1}^{[r]}(A, \mu)= \begin{cases}\left(\frac{1}{\mu(A)} \int_{A} t^{r} d \mu(t)\right)^{\frac{1}{r}} & \text { for } r \neq 0, \\ \exp \left(\frac{1}{\mu(A)} \int_{A} \ln t d \mu(t)\right) & \text { for } r=0\end{cases}
$$

Respecting the mark for integral power mean, it comes next $\mathcal{M}_{1}^{[1]}(A, \mu)=\mathcal{B}(A, \mu)$.

Corollary 4.2 Let $\mu$ be a finite measure on I. Let $B \subseteq I$ be a $\mu$-measurable set and $A \subset B$ be a bounded interval such that

$$
0<\mu(A)<\mu(B) .
$$

\section{If one of the equalities}

$$
\mathcal{M}_{1}^{[1]}(A, \mu)=\mathcal{M}_{1}^{[1]}(B, \mu)=\mathcal{M}_{1}^{[1]}(B \backslash A, \mu)
$$

is valid, then the double inequality

$$
\mathcal{M}_{1}^{[r]}(A, \mu) \leq \mathcal{M}_{1}^{[r]}(B, \mu) \leq \mathcal{M}_{1}^{[r]}(B \backslash A, \mu)
$$

holds for $r \geq 1$, at the same time as the double inequality

$$
\mathcal{M}_{1}^{[r]}(A, \mu) \geq \mathcal{M}_{1}^{[r]}(B, \mu) \geq \mathcal{M}_{1}^{[r]}(B \backslash A, \mu)
$$

holds for $r \leq 1$.

Proof The proof of corollary follows from Theorem 4.1 with functions $\varphi(t)=t$ and $\psi(t)=$ $t^{r}$ for $r \neq 0$ or $\psi(t)=\ln t$ for $r=0$.

General forms and refinements of quasi-arithmetic means can be found in [7]. 
References

1. Karlin, S, Novikoff, A: Generalized convex inequalities. Pac. J. Math. 13, 1251-1279 (1963)

2. Pavić, Z, Pečarić, J, Perić, I: Integral, discrete and functional variants of Jensen's inequality. J. Math. Inequal. 5(2), 253-264 (2011)

3. Niculescu, CP, Persson, LE: Convex Functions and Their Applications. Canadian Mathematical Society. Springer, New York (2006)

4. Sandor, J: On global bounds for generalized Jensen's inequality. Ann. Funct. Anal. 4(1), 18-24 (2013)

5. Conde, C: A version of the Hermite-Hadamard inequality in a nonpositive curvature space. Banach J. Math. Anal. 6(2), 159-167 (2012)

6. Pečarić, JE, Proschan, F, Tong, YL: Convex Functions, Partial Orderings, and Statistical Applications. Academic Press, New York (1992)

7. Mićić, J, Pavić, Z, Pečarić, J: The inequalities for quasiarithmetic means. Abstr. Appl. Anal. 2012, Article ID 203145 (2012)

doi:10.1186/1029-242X-2013-61

Cite this article as: Pavić: Convex combinations, barycenters and convex functions. Journal of Inequalities and Applications 2013 2013:61.

Submit your manuscript to a SpringerOpen ${ }^{\circ}$ journal and benefit from:

- Convenient online submission

- Rigorous peer review

- Immediate publication on acceptance

- Open access: articles freely available online

- High visibility within the field

- Retaining the copyright to your article

Submit your next manuscript at $>$ springeropen.com 